\title{
Thermodynamics of noncommutative de Sitter spacetime
}

\author{
B. Vakili, N. Khosravi ${ }^{\dagger}$ and H. R. Sepangi ${ }^{\ddagger}$ \\ Department of Physics, Shahid Beheshti University, Evin, Tehran 19839, Iran
}

October 30, 2018

\begin{abstract}
We study the effects of noncommutativity of spacetime geometry on the thermodynamical properties of the de Sitter horizon. We show that noncommutativity results in modifications in temperature, entropy and vacuum energy and that these modifications are of order of the Planck scale, suggesting that the size of the noncommutative parameter should be close to that of the Planck. In an alternative way to deal with noncommutativity, we obtain a quantization rule for the entropy. Since noncommutativity in spacetime geometry modifies the Heisenberg algebra and introduces the general uncertainty principle, we also investigate the above problem in this framework.
\end{abstract}

Keywords: de Sitter spacetime; Noncommutativity; Generalized Uncertainty Principle.

PACS numbers: 04.60.-m, 04.60.Ds, 04.60.Kz

\section{Introduction}

The black hole thermodynamics formulation, first introduced by Bekenstein [1] and a few years later by Hawking [2], is based on applying quantum field theory to the curved spacetime of a black hole. According to this formalism a black hole emits radiation with a temperature

$$
T_{B H}=\frac{\hbar \kappa}{2 \pi k_{B} c}
$$

where $\kappa$ is the surface gravity at the black hole horizon and $k_{B}$ is the Boltzmann constant. In the case of a Schwarzschild black hole the temperature can be written as

$$
T_{B H}=\frac{\hbar c^{3}}{8 \pi G k_{B} m}
$$

with an entropy, given by

$$
S_{B H}=\frac{1}{4} \frac{k_{B} c^{3}}{G \hbar} A
$$

where $A$ is the area of the black hole event horizon. The Hawking radiation of a black hole is due to the random processes in the quantum fields near the horizon. The mechanism of this thermal radiation can be explained in terms of pair creation in the gravitational potential well of the black

*email: b-vakili@sbu.ac.ir

†email: n-khosravi@sbu.ac.ir

${ }^{\ddagger}$ email: hr-sepangi@sbu.ac.ir 
hole [3]. The conclusions of the above works is that the temperature of a black hole is proportional to the surface gravity and that the area of its event horizon plays the role of its entropy. In this scenario the black hole is akin to a thermodynamical system obeying the usual thermodynamics laws, often called the laws of black hole mechanics, first formulated by Hawking [2].

In more recent times, this issue has been at the center of concerted efforts in the hope of describing and making clear various aspects of the problem still remaining unclear, for a review see [4]. With the birth of string theory [5] as a candidate for quantum gravity and loop quantum gravity [6], a new window was opened to the problem of black hole radiation. This was because the nature of black hole radiation is such that quantum gravity effects can not be neglected [7]. Motivated by sting theory, noncommutative spaces [8] and the Generalized Uncertainty Principle (GUP) [9] have been studied extensively and applied to black hole physics, leading to some modifications of the Hawking theory which were comparable with those that came from string theory and loop quantum gravity [10]-[16].

An interesting feature of the black hole thermodynamics theory is the close connections between the black hole event horizon and cosmological horizon in so far as the thermodynamical properties are concerned. This issue was first introduced by Gibbons and Hawking in [17] where they extended the black hole thermodynamics to the cosmological models with a positive (repulsive) cosmological constant. Indeed, they showed that in analogy with a black hole, the area of the event horizon in these models can play the role of entropy or lack of information on the regions inaccessible to an observer. Also, one can associate a surface gravity, $\kappa$, to the event horizon which can be interpreted as temperature. Applying the same procedure to the cosmological event horizon, they showed that laws very similar to zeroth, first and second laws of black hole mechanics are obeyed. Particulary, the particle creation with a thermal spectrum would also occur in these cosmological models, as is shown in [17] and [18] for a de Sitter model. Recently, it has been shown that the Friedmann equation describing the dynamics of an accelerated expanding universe can be obtained by applying the first law of thermodynamics to the apparent horizon of the FRW universe. This issue is not only investigated in the usual Einstein-Hilbert framework but in the case of scalar-tensor type $f(R)$ gravity theories like that of the Gauss-Bonnet and the more general Lovelock [19]-[22].

In this paper, motivated by the works done in the context of noncommutative black hole physics, we study the effects of noncommutativity on the thermodynamical properties of de Sitter spacetime as the simplest model with a cosmological horizon. As is well known from the work of Gibbons and Hawking [17], this spacetime has an apparent horizon which behaves like the black hole horizon as far as thermal radiation is concerned. Since this phenomena is described quantum mechanically, the quantum gravitational effects can not be neglected and this paves the way for our physical motivation to deal with this problem. Since there is not a unique approach in dealing with noncommutativity in such models, we consider different approaches and compare the results.

The organization of the paper is as follows: in section 2, we review the thermodynamical properties of the de Sitter space. In section 3, to introduce noncommutativity, we directly replace the coordinates with their noncommutative counterparts and obtain new thermodynamical variables which are different from their commutative version. In section 4 , we deal with noncommutative spaces in an alternative way by considering the horizon equation as a constraint and obtain a quantization rule for entropy. Section 5 deals with the same problem in the framework of GUP. Finally, we summarize the conclusions in section 6 . In what follows, we work in units where $c=\hbar=G=k_{B}=1$.

\section{Thermodynamics of the de Sitter spacetime: commutative case}

The simplest solution to Einstein equations with a cosmological constant, $R_{\mu \nu}-\frac{1}{2} R g_{\mu \nu}+\Lambda g_{\mu \nu}=$ $8 \pi T_{\mu \nu}$, is the de Sitter spacetime which is a solution of the above field equations with $T_{\mu \nu}=0$ [23]. The corresponding metric in a static form can be written as

$$
d s^{2}=-\left(1-\frac{\Lambda}{3} r^{2}\right) d t^{2}+\left(1-\frac{\Lambda}{3} r^{2}\right)^{-1} d r^{2}+r^{2}\left(d \vartheta^{2}+\sin ^{2} \vartheta d \phi^{2}\right) .
$$


This metric has an apparent singularity at $r_{h}=\sqrt{3 / \Lambda}{ }^{1}$ This singularity is like the coordinate singularity associated with horizon in the Schwarzschild spacetime, and as is well known, there are other coordinate systems for which this type of singularity is removed [24]. Thus, such a singularity is of the horizon type, meaning that the light cone tips over for $r>\sqrt{3 / \Lambda}$ and $\partial / \partial r$ becomes timelike while $\partial / \partial t$ becomes spacelike. This means that one can send signals outside the horizon through the $r_{h}=\sqrt{3 / \Lambda}$ surface but can never receive information from there, $i$. $e$. the de Sitter horizon is a one-way membrane, from inside to the outside.

As we mentioned in the introduction, the similarity between the black hole horizon and the de Sitter horizon is the main motivation to assign a temperature and an entropy to the horizon in the de Sitter space. In spite of their different nature, one being a black hole horizon and the other a cosmological one as is shown in [17], they have many of the same thermodynamical properties.

In this section, we briefly review the thermodynamics of the de Sitter horizon in a commutative spacetime with the usual canonical commutation relations prevailing, that is

$$
\left[x_{i}, x_{j}\right]=0, \quad\left[x_{i}, p_{j}\right]=i \delta_{i j}, \quad\left[p_{i}, p_{j}\right]=0 .
$$

In the classical limit the quantum mechanical commutators should be replaced by the classical Poisson brackets as $[P, Q] \rightarrow i\{P, Q\}$. As we indicated above, the de Sitter spacetime (4) has a horizon at $r_{h}=\sqrt{3 / \Lambda}$ with the following area

$$
A=r_{h}^{2} \int_{0}^{2 \pi} d \phi \int_{0}^{\pi} \sin ^{2} \vartheta d \vartheta=4 \pi r_{h}^{2}=\frac{12 \pi}{\Lambda} .
$$

This horizon is a null surface, that is, vectors normal to this surface are null. Thus, if we consider the Killing vector $\xi$, generating time translations $(\xi=\partial / \partial t)$, it is normal to the horizon $\left(\xi_{\mu} \xi^{\mu}=0\right)$. Therefore, $\xi_{\mu} \xi^{\mu}$ is constant on the horizon and thus $\nabla^{\alpha}\left(\xi_{\mu} \xi^{\mu}\right)$ is also normal to the horizon. If one normalizes $\xi$ to have unit magnitude at the origin [17], one can define a surface gravity for the horizon, such that on the horizon

$$
\nabla^{\alpha}\left(\xi_{\mu} \xi^{\mu}\right)=2 \kappa \xi^{\alpha}
$$

For a diagonal metric in spherical coordinates whose components are dependent on $r$ only, it is easy to show that

$$
\kappa=\sqrt{-\frac{1}{4} g^{r r} g^{t t}\left(\frac{\partial g_{t t}}{\partial r}\right)^{2}},
$$

evaluated at the horizon. For the de Sitter space (4), this gives

$$
\kappa=\sqrt{\frac{\Lambda}{3}}
$$

According to the Gibbons and Hawking analysis of particle creation in de Sitter spacetime [17], one can assign temperature and entropy to this spacetime, using equations (1)-(3), (6) and (9), leading to

$$
\begin{gathered}
T_{G H}=\frac{1}{\beta_{G H}}=\frac{1}{2 \pi} \sqrt{\frac{\Lambda}{3}}, \\
S_{G H}=\frac{3 \pi}{\Lambda} .
\end{gathered}
$$

This temperature is associated with an isotropic background of thermal radiation measured by a freely moving observer in de Sitter space. One can describe this thermal radiation in terms of pair creation in the gravitational potential well of the de Sitter spacetime. Outside the horizon and near it, there are particle states which are energetically allowed to create a pair of particles. One such

\footnotetext{
${ }^{1}$ We consider the positive cosmological constant only. It is clear that for a negative cosmological constant, the metric does not have any singularity. In general, models with negative cosmological constant do not have an event horizon [17].
} 
particle goes to infinity and the other can tunnel through the horizon and become absorbed by an observer in the de Sitter space. The rate of such particle emission is calculated in [17] where it is shown that the result is similar to radiation from a black body with temperature $T=\frac{1}{2 \pi} \sqrt{\Lambda / 3}$.

Applying the first law of thermodynamics

$$
d E_{G H}=T_{G H} d S_{G H},
$$

to the horizon one can evaluate the vacuum energy of the de Sitter space by integration. Using equations (10) and (11), we obtain

$$
E_{G H}=\sqrt{\frac{3}{\Lambda}}=\frac{1}{2 \pi} \beta_{G H} .
$$

There is also an alternative way to derive the above results using statistical methods. In this method, we first express the de Sitter metric in Kruskal coordinates [17]

$$
d s^{2}=\frac{3}{\Lambda}(U V-1)^{-2}\left[-4 d U d V+(U V+1)^{2}\left(d \vartheta^{2}+\sin ^{2} \vartheta d \phi^{2}\right)\right]
$$

where

$$
r=\sqrt{\frac{3}{\Lambda}}(U V+1)(1-U V), \quad \exp \left(2 \sqrt{\frac{\Lambda}{3}} t\right)=-V U^{-1} .
$$

Now, it is clear from this coordinate system that the corresponding spacetime has an imaginary period $\beta$

$$
\beta=2 \pi \sqrt{\frac{3}{\Lambda}}
$$

which is in agreement with relation (10) that gives the corresponding temperature. Now, following [25], we introduce the partition function of the (gravitational) system through the following path integral

$$
Z=\sum_{n} e^{-\beta E_{n}}=\int D[g] e^{-\hat{I}}, \quad \ln Z=-\hat{I},
$$

where the summation is over the different quantum states and $\hat{I}$ is the Euclidean action. For the de Sitter spacetime the Euclidean action can be obtained as, see [25] and [26]

$$
\hat{I}=\frac{3 \pi}{\Lambda}=\frac{1}{4 \pi} \beta_{G H}^{2}
$$

Thus, we have

$$
\ln Z=-\frac{1}{4 \pi} \beta^{2}
$$

Now, the energy and entropy of the gravitational system can be evaluated from the usual relations

$$
\bar{E}=-\frac{\partial \ln Z}{\partial \beta}, \quad S=\beta \bar{E}+\ln Z
$$

with the result

$$
\bar{E}=\frac{1}{2 \pi} \beta=\sqrt{\frac{3}{\Lambda}}, \quad S=\frac{1}{4 \pi} \beta^{2}=\frac{3 \pi}{\Lambda} .
$$




\section{Thermodynamics of de Sitter spacetime: noncommutative case}

Let us now concentrate on the noncommutativity concepts in the model described above, hoping to reach modifications which are closely related to those coming from quantum gravity or string theory.

Noncommutativity is described by a deformed product, also known as the Moyal product law between two arbitrary functions of position and momenta as

$$
\left(f *_{\alpha} g\right)(x)=\left.\exp \left[\frac{i}{2} \alpha^{a b} \partial_{a}^{(1)} \partial_{b}^{(2)}\right] f\left(x_{1}\right) g\left(x_{2}\right)\right|_{x_{1}=x_{2}=x}
$$

such that

$$
\alpha_{a b}=\left(\begin{array}{cc}
\Theta_{i j} & \delta_{i j} \\
-\delta_{i j} & 0
\end{array}\right),
$$

where the $N \times N$ matrix $\Theta$ is assumed to be antisymmetric with $2 N$ being the dimension of the phase space and represents the noncommutativity in coordinates. With this product law, the deformed commutation relations can be written as

$$
[f, g]_{\alpha}=f *_{\alpha} g-g *_{\alpha} f
$$

A simple calculation shows that

$$
\left[x_{i}, x_{j}\right]_{\alpha}=i \Theta_{i j}, \quad\left[x_{i}, p_{j}\right]_{\alpha}=i \delta_{i j}, \quad\left[p_{i}, p_{j}\right]_{\alpha}=0 .
$$

Now, consider the following transformations

$$
\hat{x}_{i}=x_{i}-\frac{1}{2} \Theta_{i j} p_{j}, \quad \hat{p}_{i}=p_{i} .
$$

It can easily be checked that if $\left(x_{i}, p_{j}\right)$ obey the usual (Poisson) commutation relations (5), then

$$
\left\{\hat{x}_{i}, \hat{x}_{j}\right\}=\Theta_{i j}, \quad\left\{\hat{x}_{i}, \hat{p}_{j}\right\}=\delta_{i j}, \quad\left\{\hat{p}_{i}, \hat{p}_{j}\right\}=0 .
$$

These Poisson brackets are the same as the Poisson bracket counterparts of relations (25). Consequently, for introducing noncommutativity, it is more convenient to work with commutation relations (27) than $\alpha$-star deformed commutation relations (25). It is important to note that the relations represented by equations (25) are defined in the spirit of the Moyal product given above. However, in the relations defined by equations $(27)$, the variables $\left(\hat{x}_{i}, \hat{p}_{j}\right)$ obey the usual commutation relations, so that the two sets of deformed and ordinary commutation relations represented by (25) and (27) should be considered as distinct.

Now, consider the noncommutative de Sitter spacetime whose metric is given by

$$
d s^{2}=-\left(1-\frac{\Lambda}{3} \hat{r} \hat{r}\right) d t^{2}+\left(1-\frac{\Lambda}{3} \hat{r} \hat{r}\right)^{-1} d \hat{r} d \hat{r}+\hat{r} \hat{r}\left(d \vartheta^{2}+\sin ^{2} \vartheta d \phi^{2}\right)
$$

where $\hat{r}$ satisfies (27). The horizon radius of the noncommutative de Sitter space can be obtained from the following condition

$$
1-\frac{\Lambda}{3} \hat{r} \hat{r}=0 .
$$

Applying the transformations (26) to this condition, the horizon of the noncommutative de Sitter space should satisfy the following relation

$$
1-\frac{\Lambda}{3}\left(x_{i}-\frac{1}{2} \Theta_{i j} p_{j}\right)\left(x_{i}-\frac{1}{2} \Theta_{i k} p_{k}\right)=0,
$$

which leads to

$$
1-\frac{\Lambda}{3}\left[r^{2}-\vec{\Theta} \cdot \vec{L}+\frac{1}{4}\left(\Theta^{2} p^{2}-(\vec{\Theta} \cdot \vec{p})^{2}\right)\right]=0
$$


where $\vec{L}=\vec{r} \times \vec{p}$ and $\Theta_{i j}=\epsilon_{i j k} \Theta_{k}$. If, as is done in [11], we take $\vec{\Theta}=(0,0, \Theta)$, (which can be done by a rotation or redefinition of coordinates), we get

$$
1-\frac{\Lambda}{3}\left[r^{2}-\Theta L_{z}+\frac{1}{4} \Theta^{2}\left(p_{x}^{2}+p_{y}^{2}\right)\right]=0 .
$$

Since the de Sitter spacetime is non-rotating, $L_{z}=0$ and by definition $\theta^{2}=\frac{1}{4}\left(p_{x}^{2}+p_{y}^{2}\right) \Theta^{2}$, we are led to the radius of noncommutative de Sitter spacetime

$$
r_{n c}=\sqrt{\frac{3}{\Lambda}-\theta^{2}}
$$

or taking terms to second order of the noncommutative parameter $\theta$, we have

$$
r_{n c}=r_{h}\left(1-\frac{1}{2} \frac{\theta^{2}}{r_{h}^{2}}\right)
$$

We see that the radius of the horizon in noncommutative case is less than its commutative counterpart. The horizon area of the noncommutative de Sitter space defined as $A_{n c}=4 \pi r_{n c}^{2}$ yields

$$
A_{n c}=A\left(1-\frac{4 \pi \theta^{2}}{A}\right) .
$$

Thus, the noncommutative entropy corresponding to the noncommutative space reads $S_{n c}=\frac{1}{4} A_{n c}$, with the result

$$
S_{n c}=S_{G H}\left(1-\frac{\pi \theta^{2}}{S_{G H}}\right) .
$$

Also, to obtain the temperature in this case we should first evaluate the noncommutative surface gravity. Extending equation (8) to the noncommutative spacetime (28), we get

$$
\kappa_{n c}=\frac{\Lambda}{3} \hat{r}
$$

which should be evaluated at the noncommutative horizon radius (33) with the result

$$
\kappa_{n c}=\frac{\Lambda}{3} \sqrt{\frac{3}{\Lambda}-\theta^{2}} .
$$

Now, using the temperature formula (1) in the noncommutative case as well, we obtain the corresponding temperature as

$$
T_{n c}=\frac{1}{2 \pi} \frac{\Lambda}{3} \sqrt{\frac{3}{\Lambda}-\theta^{2}},
$$

or, up to second order of the noncommutative parameter

$$
T_{n c}=T_{G H}\left(1-2 \pi^{2} \theta^{2} T_{G H}^{2}\right) .
$$

Finally, to calculate the vacuum energy of the noncommutative de Sitter spacetime, we assume that the first law of thermodynamics is also valid in this case as $d E_{n c}=T_{n c} d S_{n c}$. Use of equations (36) and (40) then yields

$$
d E_{n c}=d E_{G H}\left(1-\frac{1}{2} \frac{\theta^{2}}{E_{G H}^{2}}\right)
$$

or after integration

$$
E_{n c}=E_{G H}\left(1+\frac{\theta^{2}}{2 E_{G H}^{2}}\right)
$$




\section{Quantized entropy in noncommutative de Sitter spacetime}

In the previous section, to introduce noncommutativity in de Sitter spacetime we directly replaced the coordinates with their noncommutative counterparts. Subsequently, using transformation (26) we found the radius (34) for the noncommutative de Sitter space. In this section we shall deal with this issue in a different manner by considering the relation $r^{2}=r_{h}^{2}=3 / \Lambda$ as a constraint. Assuming that this constraint also holds in the noncommutative space in the form $\hat{r} \hat{r}=r_{h}^{2}$, we proceed to quantize it through the use of transformation (26) and use of the replacement $p_{i} \rightarrow-i \partial_{i}$, that is

$$
\left(x_{i}+\frac{i}{2} \Theta_{i j} \partial_{j}\right)\left(x_{i}+\frac{i}{2} \Theta_{i k} \partial_{k}\right) \psi(\vec{x})=r_{h}^{2} \psi(\vec{x}),
$$

where $\psi(\vec{x})$ is a wave function which can be annihilated by the constraint $\hat{r} \hat{r}-r_{h}^{2}=0$. As before, taking $\Theta_{i j}=\epsilon_{i j k} \Theta_{k}$ and $\vec{\Theta}=(0,0, \Theta)$, we obtain the following eigenvalue equation

$$
\left[-\frac{1}{2}\left(\frac{\partial^{2}}{\partial x^{2}}+\frac{\partial^{2}}{\partial y^{2}}\right)+\frac{2 i}{\Theta}\left(x \frac{\partial}{\partial y}-y \frac{\partial}{\partial x}\right)+\frac{2}{\Theta^{2}}\left(x^{2}+y^{2}+z^{2}\right)\right] \psi(\vec{x})=\frac{2 r_{h}^{2}}{\Theta^{2}} \psi(\vec{x}),
$$

which is a Schrödinger like equation for a charged particle with unit mass moving in a magnetic field. Indeed, for a uniform magnetic field $\vec{B}=(0,0, B)$, the Schrödinger equation for a particle with unit mass and charge $q$ reads

$$
\left[-\frac{1}{2}\left(\frac{\partial^{2}}{\partial x^{2}}+\frac{\partial^{2}}{\partial y^{2}}+\frac{\partial^{2}}{\partial z^{2}}\right)+\frac{q B}{2} i\left(x \frac{\partial}{\partial y}-y \frac{\partial}{\partial x}\right)+\frac{q^{2} B^{2}}{8}\left(x^{2}+y^{2}\right)\right] \psi(\vec{x})=E \psi(\vec{x}) .
$$

As is well known from ordinary quantum mechanics the motion of such a particle along the $z$-axis is a uniform motion $z=v_{0 z} t+z_{0}$ and the corresponding energy eigenvalues are the Landau levels $E_{n}=\frac{1}{2} v_{0 z}^{2}+q B\left(n+\frac{1}{2}\right)$. Now, if we take the constant $v_{0 z}$ to be zero, the particle will move in the plane $z=z_{0}$. In this case the wave function representing the particle is independent of $z$ and the

term $-\frac{1}{2} \frac{\partial^{2} \psi}{\partial z^{2}}$ will be removed from equation (45). Therefore, comparison of equations (45) and (44) show that we can identify $q B$ with $\frac{4}{\Theta}$ and bearing in the mind the Landau levels, we find

$$
\frac{2}{\Theta^{2}}\left(r_{h}^{2}-z_{0}^{2}\right)=\left(n+\frac{1}{2}\right) \frac{4}{\Theta} .
$$

To achieve the correct result in the limit $\Theta \rightarrow 0$ we take $z_{0}=\sqrt{3 / \Lambda}$ and thus

$$
r_{h}^{2}=\frac{3}{\Lambda}+2 \Theta\left(n+\frac{1}{2}\right)
$$

Also, the entropy of the noncommutative de Sitter spacetime, $S=A / 4=4 \pi r_{h}^{2} / 4$, can be written as

$$
S=\frac{3 \pi}{\Lambda}+2 \pi \Theta\left(n+\frac{1}{2}\right) .
$$

Therefore, we see that introduction of noncommutativity in this approach yields certain quantization rules for the horizon radius and entropy of the de Sitter spacetime.

\section{Thermodynamics of de Sitter spacetime with GUP}

Let us now study the model described above in the framework of GUP. Here, the Heisenberg algebra is modified by the addition of small corrections to the commutation relations. The resulting modifications implies a minimum position uncertainty of order of the Planck length suggested by quantum gravity and string theory. This framework is thus suitable to the study of the de Sitter spacetime at the quantum level. 
We start with the GUP defined as

$$
\delta x \delta p \geq \hbar+l_{p}^{2} \frac{(\delta p)^{2}}{\hbar}
$$

where $l_{p}=\sqrt{G \hbar / c^{3}}$ is the Planck length. Thus, uncertainty formula leads to ${ }^{2}$

$$
\delta p=\frac{\delta x}{2 l_{p}^{2}}\left[1-\sqrt{1-\frac{4 l_{p}^{2}}{(\delta x)^{2}}}\right] .
$$

Now, applying the uncertainty principle to the horizon radius results in

$$
T \sim \frac{\delta p}{2 \pi}
$$

Note that the same result is also valid for temperature of a black hole, see [1]. Taking $\delta x=r_{h}=\sqrt{3 / \Lambda}$ and use of equation (50) leads to the modified temperature based on GUP

$$
T_{G U P}=\frac{1}{4 \pi l_{p}^{2}} \sqrt{\frac{3}{\Lambda}}\left[1-\sqrt{1-\frac{4 \Lambda l_{p}^{2}}{3}}\right],
$$

where up to second order in Planck length we have

$$
T_{G U P}=T_{G H}\left(1+4 \pi^{2} l_{p}^{2} T_{G H}^{2}\right) .
$$

To evaluate the entropy of the de Sitter space in the GUP framework we do as follows. Assume that because of particle radiation described in [17], the area $A=4 \pi r_{h}^{2}$ of the horizon changes as

$$
d A=8 \pi r_{h} d r_{h}
$$

where $r_{h}=\sqrt{3 / \Lambda}$. During this process the energy of the de Sitter spacetime changes as $\delta E$ such that

$$
\delta A=\frac{24 \pi}{\sqrt{3 \Lambda}} \delta E
$$

where we have used relation (13). Now, the uncertainty principle for radiated particles implies $\delta E \sim \delta p \sim 1 / \delta x$ and thus

$$
\delta A=\frac{24 \pi}{\sqrt{3 \Lambda}} \delta p=\frac{24 \pi}{\sqrt{3 \Lambda}} \frac{1}{\delta x} .
$$

Extending this relation to the GUP case, we are led to

$$
\delta A_{G U P}=\frac{24 \pi}{\sqrt{3 \Lambda}} \frac{\delta x}{2 l_{p}^{2}}\left[1-\sqrt{1-\frac{4 l_{p}^{2}}{(\delta x)^{2}}}\right],
$$

where keeping terms up to fourth order in Planck length yields

$$
\delta A_{G U P}=\frac{24 \pi}{\sqrt{3 \Lambda}} \frac{1}{\delta x}\left[1+\frac{l_{p}^{2}}{(\delta x)^{2}}+\frac{2 l_{p}^{4}}{(\delta x)^{4}}\right] .
$$

Now, taking $\delta x=r_{h}=\sqrt{A / 4 \pi}$, we get

$$
d A_{G U P}=\left(1+\frac{4 \pi l_{p}^{2}}{A}+\frac{32 \pi^{2} l_{p}^{4}}{A^{2}}\right) d A .
$$

\footnotetext{
${ }^{2}$ Although in units adopted here the Planck length is equal to unity, we retain it in the ensuing formulae to follow the role it plays which is an important one.
} 
Integration then yields

$$
A_{G U P}=A+4 \pi l_{p}^{2} \ln A-32 \pi^{2} l_{p}^{4} \frac{1}{A} .
$$

Now, by extending the entropy formula $S=A / 4$ to the GUP case we obtain an expression for the entropy of the de Sitter space in the GUP framework

$$
S_{G U P}=S_{G H}+\pi l_{p}^{2} \ln S_{G H}-2 \pi^{2} l_{p}^{4} \frac{1}{S_{G H}}+\ldots+C,
$$

where $C$ is a constant proportional to $l_{p}^{2}$. Finally, to obtain the energy of the de Sitter spacetime based on GUP, we assume that the first law of thermodynamics is also valid in this case : $d E_{G U P}=$ $T_{G U P} d S_{G U P}$. Now, using equations (53) and (61), we find an expression for energy after integration as

$$
E_{G U P}=E_{G H}\left(1-\frac{2 l_{p}^{2}}{E_{G H}^{2}}-\frac{l_{p}^{4}}{E_{G H}^{4}}\right) .
$$

\section{Conclusions}

In this paper we have studied the effects of noncommutativity on the thermodynamical properties of de Sitter spacetimes. The physical relevance of such attempts is that de Sitter spacetimes radiate a thermal spectrum which behaves like the Hawking radiation for black holes and should be described quantum mechanically and thus includes quantum gravitational effects, indicating that the spacetime is noncommutative. We have used this analogy with the black hole radiation formulae for temperature and entropy to obtain the temperature of de Sitter spacetimes as a radiating thermodynamical system and, therefore, assigned an entropy to it. Assuming that this system obeys the first law of thermodynamics, we have found the vacuum energy of de Sitter spacetimes. We then introduced noncommutativity in the spacetime metric by replacing the coordinates with their noncommutative counterparts. This led to new relationships for radius of the horizon, temperature, entropy and energy of the system, all showing similar results akin to those of the commutative case but incorporating some correction terms. From the noncommutative thermodynamical variables, we see that the horizon radius, temperature and entropy of the de Sitter space are decreased, while its energy increased.

The noncommutative coordinates was subsequently dealt with in an alternative way. In this approach one can obtain the horizon radius as a constraint equation which also holds in the noncommutative case. Here, we have seen that the noncommutativity effect resembles the presence of a magnetic field. Quantizing this constraint led us to a quantization rule for the entropy of de Sitter spacetime. We have also investigated the problem by applying GUP on the de Sitter horizon. In this framework we have obtained the thermodynamical variables with some different correction terms. Particulary, the correction term in the entropy formula is a logarithmic term which is a known correction term in quantum black hole physics. This is in contrast to the previous result for noncommutative case in which the first order correction term is a constant shift in (36).

An important issue in our analysis is that the different approaches dealing with noncommutativity in a phenomenological framework, result in different correction terms to thermodynamical quantities of the de Sitter spacetime. For example $E_{n c}$ in equation (42) is larger than $E_{G H}$ while $E_{G U P}$ in equation (62) diminishes with respect to $E_{G H}$. This difference in the sign of the correction terms also appears in the black hole thermodynamics when one considers the quantum gravitational effects $[27,28]$. As is indicated in [28] "the sign of a logarithmic term can be understood as either related to the missing information (if it is positive) or the increase of information (if it is negative), depending on whether the horizon area is fixed or not". A complete answer to this still open question is not possible until a full theory of quantum gravity emerges, a theory which presently does not exist in a complete form. 


\section{References}

[1] J. D. Bekenstein, Phys. Rev. D 7 (1973) 2333

J. D. Bekenstein, Phys. Rev. D 9 (1974) 3292

[2] J. M. Bardeen, B. Carter and S. W. Hawking, Commun. Math. Phys. 31 (1973) 161

S. W. Hawking, Commun. Math. Phys. 43 (1975) 199

G. W. Gibbons and S. W. Hawking, Phys. Rev. D 15 (1977) 2752

[3] N. D. Birrell and P. C. W. Davies, Quantum fields in curved space (Cambridge University Press, UK, 1982)

[4] T. Padmanabhan, Phys. Rep. 406 (2005) 49

[5] N. Seiberg and E. Witten, J. High Energy Phys. JHEP 09 (1999) 032

G. T. Horowitz, New J. Phys. 7 (2005) 201 (gr-qc/0410049)

[6] A. Ashtekar, New J. Phys. 7 (2005) 198 (gr-qc/0410054)

[7] S. Mukherji and S. S. Pal, J. High Energy Phys. JHEP 0205 (2002) 026 (hep-th/0205164)

A. Ashtekar and K. Krasnov, in Black holes, Gravitational Radiation and the Universe, edited by B. Iyer and B. Bhawal, (Kluwer Dodrecht, 1999) 149 (gr-qc/9804039)

[8] H. S. Snyder, Phys. Rev. 71 (1947) 38

[9] G. Veneziano, EuroPhys. Lett. 2 (1986) 199

D. Amati, M. Ciafaloni and G. Veneziano, Phys. Lett. B 216 (1989) 41

[10] P. Nicolini, A. Smailagic and E. Spallucci, Phys. Lett. B 632 (2006) 547, (gr-qc/0510112)

[11] F. Nasseri, Int. J. Mod. Phys. D 15 (2006) 1113, (hep-th/0508122)

F. Nasseri, Gen. Rel. Grav. 37 (2005) 2223, (hep-th/0508051)

[12] K. Nozari and B. Fazlpour, Mod. Phys. Lett. A 22 (2007) 2917 (hep-th/0605109)

K. Nozari and S. H. Mehdipour, Int. J. Mod. Phys. A 21 (2006) 4979 (gr-qc/0511110)

[13] P. Nicolini, J. Phys. A 38 (2005) L631 (hep-th/0507266)

[14] F. Scardigli, Phys. Lett. B 452 (1999) 39 (hep-th/9904025)

[15] Z. Hai-Xia, L. Huai-Fan, H. Shuang-Qi and Z. Ren, Generalized uncertainty principle and correction value to the black hole entropy, (gr-qc/0608023)

[16] Y. S. Myung, Y.-W. Kim and Y.-J. Park, Phys. Lett. B 645 (2007) 393, (gr-qc/0609031)

[17] G. W. Gibbons and S. W. Hawking, Phys. Rev. D 15 (1977) 2738

[18] Q.-Q. Jiang, Class. Quantum Grav. 24 (2007) 4391 (0705.2068 [hep-th])

[19] B. Wang, Y. Gong and E. Abdalla, Phys. Rev. D 74 (2006) 083520, (gr-qc/0511051)

[20] R.-G. Cai and S. P. Kim, J. High Energy Phys. JHEP 0502 (2005) 050, (hep-th/0501055)

[21] M. Akbar and R.-G. Cai, Phys. Lett. B 635 (2006) 7, (hep-th/0602156)

[22] M. Akbar and R.-G. Cai, Phys. Rev. D 75 (2007) 084003, (hep-th/0609128)

[23] W. de Sitter, Mon. Not. Roy. Astron. Soc. 78 (1917) 3 
[24] S. W. Hawking and G. F. R. Ellis, Large Scale Structure of Spacetime (Cambridge University Press, New York, 1973)

[25] S. W. Hawking, in General Relativity: An Einstein Centerary Survey, edited by S. W. Hawking and W. Israel, (Cambridge University Press, 1979)

[26] B.-B. Wang and L. Liu, The statistic thermodynamical entropy of de Sitter space and it's 1-loop correction (gr-qc/0105091)

[27] G. Amelino-Camelia et al, Int. J. Mod. Phys. D 13 (2004) 2337

G. Amelino-Camelia, Nature 410 (2001) 1065

J. Magueijo and L. Smolin, Phys. Rev. D 67 (2003) 044017

G. Amelino-Camelia, Int. J. Mod. Phys. D 11 (2002) 35

K. Nozari and A. S. Sefidgar, Phys. Lett. B 635 (2006) 156 (gr-qc/0601116)

K. Nozari and A. S. Sefidgar, Gen. Rel. Grav. 39 (2007) 501 (gr-qc/0606046)

[28] C. Kiefer and G. Kolland, Gen. Rel. Grav. DOI 10.1007/s10714-008-0609-5 (0707.0615 [gr-qc]) 